\title{
An embarrassment of peptides
}

\author{
Molecular biology is revealing an extraordinary array of new, potential hormones. \\ The problem is how to determine their physiological functions, if any.
}

As molecular biology sweeps through pastures new, it tends to generate a mixture of despair, excitement and frustration in roughly equal parts. The despair is of those who see the limelight shifting to the molecular upstarts. The excitement stems from the amount of new information that is generated in short order by the new techniques. And the frustration comes in trying to translate the molecular information back into physiological terms.

So much was evident from two recent meetings on neuroscientific topics where the molecular approach held sway. First was a small gathering in Hamburg* and then this year's major symposium at Cold Spring Harbor, which will be reported in a later issue of Nature.

The Hamburg meeting illustrated just how effective the molecular approach is by comparison with its predecessors in establishing the structure of the precursor molecules from which neuropeptides/hormones (the distinction is ever more blurred) are derived. Many years ago it became clear that peptides are cleaved from much larger precursors - the erstwhile "big" or even "big, big" forms of the peptide. But to establish the structure of a precursor was a long, hard grind involving endless analytical biochemistry combined with radioimmunoassays and biological assays of countless samples.

Even when a precursor emerged from the quagmire, there was always the question of whether it was the original precursor or merely an intermediate. Molecular biology has turned the tables. The approach now is to home in on the messenger RNA for what is, by definition, the ultimate precursor, convert it into DNA and sequence it. A moment's consultation with the genetic code then allows the deduction of the amino code acid sequence of the precursor.

What emerges, invariably, is that the sequence of the known peptide is flanked on one or both sides by other peptides. Sometimes the existence of the other peptides is already known. For example, Dietmar Richter's laboratory has shown that in its precursor, arginine-vasopressin is followed by neurophysin II which was already known to be somewhere in the precursor. And neurophysin II is followed by a third peptide that was already known as a consti-

* Biochemical and Clinical Aspects of Neuropeptides: Synthesis, Processing and (icne Structure". 29 May-2 Junc, Hambury-Blankinsece. tuent of the pituitary but not as a molecular confederate of arginine-vasopressin and neurophysin.

More frequently, previously unknown peptides are revealed by sequencing a precursor messenger RNA. For example, mouse nerve growth factor is preceded by three new potential peptides (Nature 302, 538; 1983). And, in Hamburg, both William Rutter and Axel Ullrich described the existence, to judge mainly by a repeated cluster of regularly spaced cysteine residues, of about eight epidermal growth factor-like peptides preceeding the factor itself in its huge precursor (see p. 722 of this issue).

The excitement generated by such sequencing lies not only in the potential discovery of new peptides with biological function. For once cloned precursor DNA becomes available, it can be used to scan tissues for the presence of its equivalent messenger RNA by the technique of in situ hybridization. Olivier Civelli described in Hamburg how that technique had been used to detect messenger RNA from the proopiomelanocortin gene in several areas of the rat brain. Such an approach, still in its infancy, promises to clear up many a controversy generated by claims of the synthesis of a peptide in an organ, tissue, cell or neurone based only on its presence there (and often detected by inadequate means). Only if the relevant messenger RNA is also present can it be certain that the peptide has been synthesized in situ and not imported from elsewhere.

Some would go further and argue that the presence of messenger RNA for a peptide precursor is tantamount to proof that the peptide will be present, given that messenger RNA is seldom made when not needed and tends not to stay around for long once made. That, however, is to push the molecular biological approach too far because even if the precursor is made from the messenger, it cannot automatically be assumed that production of a given peptide follows.

That is where the frustration engendered by the molecular approach begins. Tempting though it is to assume that the numerous potential peptides spotted in a precursor sequence are really produced, proof is another matter. There are, however, strong clues in the presence of potential cleavage sites in precursor sequences; indeed, it is essentially by the cleavage sites that the presence of discrete, new peptide moieties is recognized. Com- monly the cleavage site is a pair of basic amino acids but it is clear that not all such pairs are cleaved and that less easily identifiable sites can be cleaved. Moreover, there are several well documented ways in which a cloven peptide can be further processed. Reliably predicting the end products of a precursor is therefore no child's game.

Predicting the function of the presumed end products is almost impossible. Who is to say that the second gastrin-like peptide in the gastrin precursor, the two extra glucagon-like peptides in the glucagon precursor or the eight vaguely similar peptides that precede epidermal growth factor have any function? Perhaps they are evolutionary debris. Quite probably they bear sufficient resemblance to the "authentic" peptide to exhibit some activity in any appropriate biological test. But does that imply a real function?

With peptides that bear no relationship to any other, the problem is where even to start. The number of such peptides is increasing rapidly through molecular biology but the problem, of course, predates the technique. At Hamburg, for example, Michel Chretien could do no better than to attribute some mitogenic activity to a large new pituitary peptide isolated and partially sequenced by classic means. And Chica Schaller was at a loss to explain the presence and function in mammalian brain and gastrointestinal tract of the same small peptide that she has painstakingly shown to be responsible for promoting the growth of Hydra head.

So how can the functions of peptides best be determined? One possibility is to turn to lowly organisms like the sea slug, Aplysia, or the fruit fly, Drosophila, which have several advantages, not least that they are amenable to molecular genetics. For those whose curiosity extends to mammals, current approaches to the determination of function are crude. One is to inject the peptide into animals or on to cells simply to observe the results. Another is to test the effects of injecting antibodies against the peptide.

The proper interpretation of that type of experiment and refinements of technique will depend upon the unfashionable skills of physiology. Immensely attractive though molecular biology must be to young scientists, we cannot afford to be without a new generation of bright physiologists able to pick up from where the molecular approach runs out. Peter Newmark 